\title{
Large scale dynamic model reconstruction for the central carbon metabolism of Escherichia coli
}

\author{
Rafael S. Costa, Daniel Machado, Isabel Rocha, Eugénio C. Ferreira \\ IBB-Institute for Biotechnology and Bioengineering, Centre of Biological Engineering, \\ University of Minho, Campus de Gualtar, P-4710-057 Braga, Portugal \\ \{rafacosta,dmachado,irocha,ecferreira\}@deb.uminho.pt
}

\begin{abstract}
The major objective of metabolic engineering is the construction of industrially relevant microbial strains with desired properties. From an engineering perspective, dynamic mathematical modeling to quantitatively assess intracellular metabolism and predict the complex behavior of living cells is one of the most successful tools to achieve that goal. In this work, we present an expansion of the original E. coli dynamic model [1], which links the acetate metabolism and tricarboxylic acid cycle (TCA) with the phosphotransferase systems, the pentose-phosphate pathway and the glycolysis system based on mechanistic enzymatic rate equations. The kinetic information is collected from available database and literature, and is used as an initial guess for the global fitting. The results of the numeric simulations were in good agreement with the experimental results. Thus, the results are sufficiently good to prompt us to seek further experimental data for comparison with the simulations.
\end{abstract}

Keywords: dynamic modeling, systems biology, E. coli, TCA cycle and acetate metabolism, enzyme kinetics

\section{Introduction}

In the last years a variety of mathematical models have been published in the literature for microorganisms with successful industrial applications [2, 3]. However, when a more detailed investigation of the complexity of genetic and metabolic pathways is desired, models that incorporate intracellular metabolism are necessary. One of the most suitable large-scale cell models are the models that account for dynamics at the enzyme and metabolite levels. Dynamic modeling provides a basis for prediction under various perturbations and can be applied to enhance the yield of desired products [4]. Dynamic models of microbial organisms are not a new issue and several works for $E$. coli are reported in the literature [5, 6]. However, in all these models, parts of the metabolic pathways are extremely simplified or omitted and more information is necessary to incorporate key metabolites and elucidate regulations of branched biosynthetic pathways. The central carbon metabolism plays essential roles in the cell, providing energy metabolism and precursors for biosynthetic reactions.

The initial point of our work is the large scale stationary model of Chassagnole et al. [1] that deals with the metabolic network of the central carbon metabolism (phosphotransferase system, glycolysis and pentose phosphate pathway) of E. coli. 


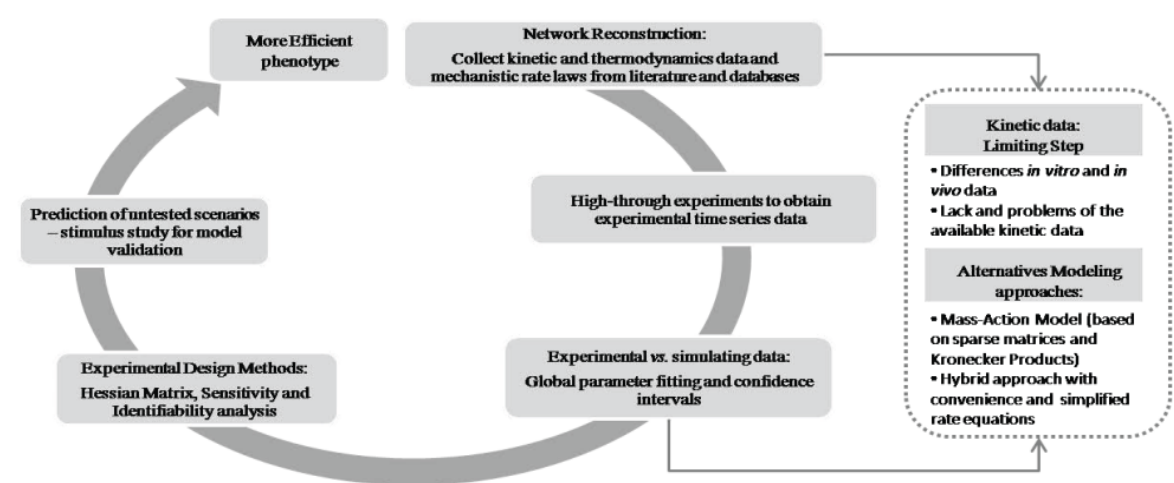

Figure 1. Schematic representation of our strategy to develop the E. coli dynamic model.

This original model was modified to introduce the TCA cycle, the glyoxylate bypass and the acetate metabolism.

In the last years vast kinetic information has been accumulated for E. coli [7, 8], however the next logical step, which consists on the integration this information, so far has been lacking. Therefore, in vitro kinetic information available in databases and literature was collected and replaced in the reconstructed model of Chassagnole et al. [1]. This reconstructed model was then used to test the consistency between the in vivo system and the parameters of the isolated enzymes (data not shown), prior to its use as an initial guess for the global parameters fitting. Model validation was also performed through comparison with experimental data. In this contribution, we give a brief overview of the project - large-scale dynamic model construction of E. coli which is now being developed in our group (see Fig. 1). In addition, results of the E. coli dynamic model reconstruction based on mechanistic rate equations are presented.

\section{Development of the Reconstructed E. coli Dynamic Model and Simulation Experiments}

The present model is based on a previous metabolic part of E. coli central metabolism model proposed by Chassagnole et al. [1]. The model was modified based on reversible Michaelis-Menten kinetics with non competing product-substrate couples for most enzymes to represent the TCA cycle, glyoxylate bypass and the main pathways involved in the acetate metabolism (phosphate acetyltransferase (PTA, EC 2.3.1.8) and acetate kinase (ACKA, EC 2.7.2.1)). However when a specific enzyme had been characterized in the literature with respect to the kinetic structure, then this type was used, such as, for isocitrate lyase (ICL, 4.1.3.1) [9], and PTA [10]. This reconstructed model consists in a total of 37 metabolites and 44 reactions (31 reversible). The biochemical network is based on publicly E. coli database EcoCyc [11] and the reaction branching from $\alpha$-ketoglutarated for precursor biosynthesis was added as described by Zhao et al. [12]. On the other hand, the in vitro kinetic parameters used in the model (maximal reaction rates, Michaelis-Menten constants, inhibition and equilibrium constants) 

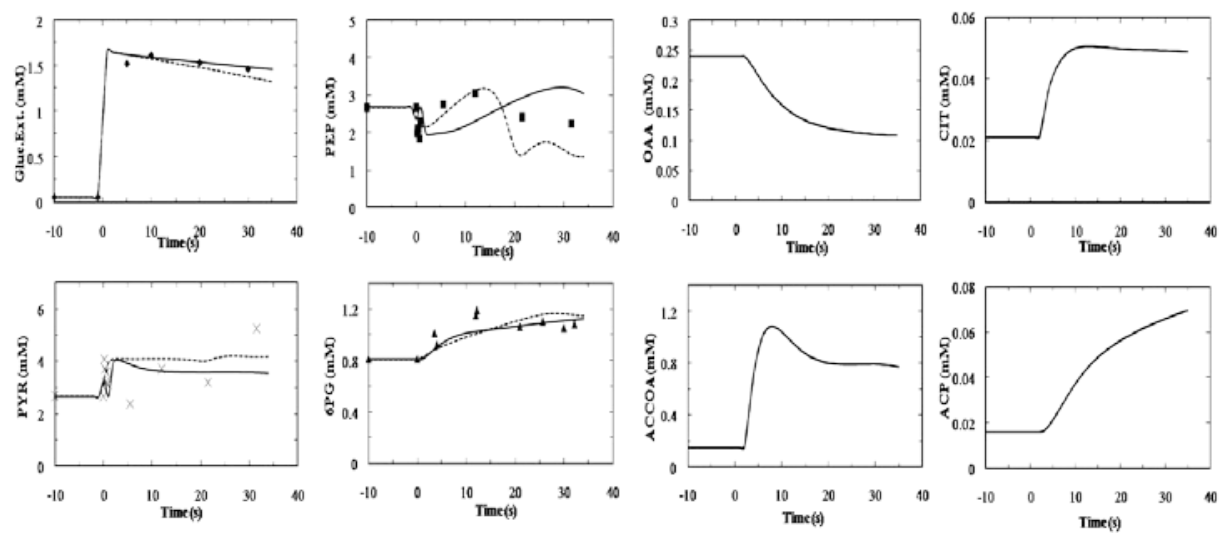

Figure 2. Comparison between simulated (dashed and solid lines for the original and reconstructed model, respectively) and experimental time-series data (symbols) for: extracellular glucose, Gluc. Ext. (•), glucose-1-phosphate, PEP (•), pyruvate, PYR (×), 6-phosphogluconate, 6PG ( $(\mathbf{4})$, after a glucose pulse at time 0 seconds. For the new metabolites Oxaloacetate (OAA), Acetyl-CoA (ACCOA), Citrate (CIT) and Acetyl-phosphate (ACP) only simulation results for the reconstructed model are shown.

of the rate equations are derived from the literature [13-15] and accessible databases $[7,8,16]$. The steady-state concentrations of the metabolites not measured for the integration as initial conditions are taken from literature $[17,18]$ and estimated from a near-equilibrium assumption with the respective equilibrium constants for each reaction from thermodynamic data [19]. The kinetic parameters were then adjusted to fit the model to the measured metabolites concentrations during the steady state in an available pathway modeling tool [20].

The parts of representative results of simulation (with both original and reconstructed model) and measured time series for metabolites concentration after a glucose pulse are shown in Figure 2. The discrepancies in metabolites concentration between experimental data and predicted simulated values from the reconstructed model were relatively small. These results indicated that the reconstructed model successfully reproduces the experimental data, but more experimental data are necessary for their validation.

\section{Concluding remarks}

In this work we developed a dynamic model of the central carbon metabolism that is based on a previous model from Chassagnole et al. [1] but was expanded by introducing the TCA cycle, glyoxylate bypass and acetate metabolism. The applicability of the model is certainly limited by the approximations made, but the good agreements of the simulation results are sufficiently encouraging to prompt us to seek further experimental data for comparison. 
Acknowledgments. Rafael S. Costa would like to thank Fundação para a Ciência e Tecnologia for providing the grant SFRH/BD/25506/2005. The authors thank Dr. C. Chassagnole, which provided the experimental data set.

\section{References}

[1] C. Chassagnole, N. Noisommit-Rizzi, J. W. Schmid, K. Mauch and M. Reuss, Dynamic modeling of the central carbon metabolism of Escherichia coli. Biotechnology and Bioengineering 79, 53-73 (2002).

[2] K. Dairaku, E. Izumoto, H. Morikawa, S. Shioya and T. Takamatsu, Optimal quality control of Baker's yeast fed-batch culture using population dynamics. Biotechnol Bioeng 24, 2661-2674 (1982).

[3] H. W. Blanch, Microbial growth kinetics. Chem Eng Commun 8, 181-211 (1981).

[4] A. K. Gombert and J. Nielsen, Mathematical modelling of metabolism. Current Opinion in Biotechnology 11, 180-186 (2000).

[5] J. D. Varner, Large-scale prediction of phenotype: Concept. Biotechnology and Bioengineering 69, 664-678 (2000).

[6] C. Chassagnole, B. Rais, E. Quentin, D. A. Fell and J. P. Mazat, An integrated study of threoninepathway enzyme kinetics in Escherichia coli. Biochemical Journal 356, 415-423 (2001).

[7] U. Wittig, M. Golebiewski, R. Kania, O. Krebs, S. Mir, A. Weidemann, S. Ansteins, J. Saric and I. Rojas, SABIO-RK: Integration and curation of reaction kinetic data. Lecture Notes in Bioinformatics 4075, 94-103 (2006).

[8] S. Sundararaj, A. Guo, B. Habibi-Nazhad, M. Rouani, P. Stothard, M. Ellison and D. S. Wishart, The CyberCell Database (CCDB): a comprehensive, self-updating, relational database to coordinate and facilitate in silico modeling of Escherichia coli. Nucleic Acids Research 32, D293D295 (2004).

[9] B. M. Bakker, P. A. M. Michels, F. R. Opperdoes and H. V. Westerhoff, Glycolysis in bloodstream form Trypanosoma brucei can be understood in terms of the kinetics of the glycolytic enzymes. Journal of Biological Chemistry 272, 3207-3215 (1997).

[10] J. Henkin and R. H. Abeles, Evidence against an Acyl-enzyme intermediate in reaction catalyzed by Clostridial phosphotransacetylase. Biochemistry 15, 3472-3479 (1976).

[11] P. Karp, M. Riley, M. Saier, Paulsen I.T., S. M. Paley and A. Pellegrini-Toole, The EcoCyc and MetaCyc databases. Nucleic Acids Research 28, 56-59 (2000).

[12] J. Zhao and K. Shimizu, Metabolic flux analysis of Escherichia coli K12 grown on C-13-labeled acetate and glucose using GG-MS and powerful flux calculation method. Journal of Biotechnology 101, 101-117 (2003).

[13] J. Tian, R. Bryk, M. Itoh, M. Suematsu and C. Nathan, Variant tricarboxylic acid cycle in Mycobacterium tuberculosis: Identification of alpha-ketoglutarate decarboxylase. Proceedings of the National Academy of Sciences of the United States of America 102, 10670-10675 (2005).

[14] M. H. N. Hoefnagel, M. J. C. Starrenburg, D. E. Martens, J. Hugenholtz, M. Kleerebezem, I. I. Van Swam, R. Bongers, H. V. Westerhoff and J. L. Snoep, Metabolic engineering of lactic acid bacteria, the combined approach: kinetic modelling, metabolic control and experimental analysis. Microbiology-Sgm 148, 1003-1013 (2002).

[15] K. Walsh and D. E. Koshland, Branch Point Control by the Phosphorylation State of Isocitrate Dehydrogenase - A Quantitative Examination of Fluxes During A Regulatory Transition. Journal of Biological Chemistry 260, 8430-8437 (1985).

[16] I. Schomburg, A. Chang and D. Schomburg, BRENDA, enzyme data and metabolic information. Nucleic Acids Research 30, 47-49 (2002).

[17] N. Ishii, K. Nakahigashi, T. Baba, M. Robert, T. Soga, A. Kanai, T. Hirasawa, M. Naba, K. Hirai, A. Hoque, P. Y. Ho, Y. Kakazu, K. Sugawara, S. Igarashi, S. Harada, T. Masuda, N. Sugiyama, T. Togashi, M. Hasegawa, Y. Takai, K. Yugi, K. Arakawa, N. Iwata, Y. Toya, Y. Nakayama, T. Nishioka, K. Shimizu, H. Mori and M. Tomita, Multiple high-throughput analyses monitor the response of E-coli to perturbations. Science 316, 593-597 (2007). 
[18] M. A. Hoque, H. Ushiyama, M. Tomita and K. Shimizu, Dynamic responses of the intracellular metabolite concentrations of the wild type and pykA mutant Escherichia coli against pulse addition of glucose or NH3 under those limiting continuous cultures. Biochemical Engineering Journal 26, 38-49 (2005).

[19] R. N. Goldberg, Y. B. Tewari and T. N. Bhat, Thermodynamics of enzyme-catalyzed reactions - $a$ database for quantitative biochemistry. Bioinformatics 20, 2874-2877 (2004).

[20] S. Hoops, S. Sahle, R. Gauges, C. Lee, J. Pahle, N. Simus, M. Singhhal, L. Xu, P. Mendes and U. Kummer, COPASI - a COmplex PAthway SImulator. Bioinformatics 22, 3067-3074 (2006). 